International Journal of Pure and Applied Mathematics

Volume 94 No. 4 2014, 537-540

ISSN: $1311-8080$ (printed version); ISSN: 1314-3395 (on-line version)

url: http://www.ijpam.eu

doi: http://dx.doi.org/10.12732/ijpam.v94i4.9

ijpam.eu

\title{
A POINTWISE NEGATIVE BINOMIAL APPROXIMATION FOR RANDOM SUMS OF GEOMETRIC RANDOM VARIABLES
}

\author{
K. Teerapabolarn \\ Department of Mathematics \\ Faculty of Science \\ Burapha University \\ Chonburi, 20131, THAILAND
}

\begin{abstract}
We determine a pointwise bound for the point metric between the distribution of random sums of independent geometric random variables and an appropriate negative binomial distribution. Two examples have been given to illustrate the result obtained.
\end{abstract}

AMS Subject Classification: 62E17, 60F05, 60G05

Key Words: geometric random variable, negative binomial approximation, point metric, random sums

\section{Introduction}

Let $X_{1}, X_{2}, \ldots$ be a sequence of independent geometric random variables, each with $P\left(X_{i}=k\right)=p_{i} q_{i}^{k}, k=0,1, \ldots$, where $q_{i}=1-p_{i}$. Let $S_{N}=\sum_{i=1}^{N} X_{i}$, where $N$ is a non-negative integer-valued random variable and independent of the $X_{i}$ 's. The random summands is usually called random sums. Let $\mathrm{s}_{n}(x)$ be the probability function of $S_{n}$ and $\mathrm{NB}_{n, p}(x)$ the negative binomial probability function with parameters $n$ and $p$, where $x \in \mathbb{N} \cup\{0\}$. For $N=n \in \mathbb{N}$ is fixed, by applying [3], we can have a pointwise bound for the point metric between

Received: March 26, 2014

(c) 2014 Academic Publications, Ltd. url: www.acadpubl.eu 
two such probability functions in the form of

$$
\left|\mathrm{S}_{n}(x)-\mathrm{NB}_{n, p}(x)\right| \leq\left(\sum_{i=1}^{n} \frac{q_{i}^{2}}{p_{i}}-\frac{n q^{2}}{p}\right) \min \left\{1, \frac{1}{\sqrt{2 n q e}}\right\},
$$

where $p=1-q=\frac{1}{n} \sum_{i=1}^{n} p_{i}$. Let $\widehat{n}=E(N)$ and $\widehat{q}=1-\widehat{p}=\frac{\lambda}{\widehat{n}}$, where $\lambda=E\left(\lambda_{N}\right)=E\left(\sum_{i=1}^{N} q_{i}\right)$. In this study, we focus on determining a pointwise bound for $\left|\mathrm{S}_{N}(x)-\mathrm{NB}_{\widehat{n}}, \widehat{p}(x)\right|$, which is in Section 2. In Section 3, we give two examples to illustrate the main result. The conclusion of this study is in the last section.

\section{Result}

The following theorem presents a pointwise bound for the point metric between $\mathrm{S}_{N}(x)$ and $\mathrm{NB}_{\widehat{n}, \widehat{p}}(x)$.

Theorem 2.1. For $x \in \mathbb{N}, \lambda_{N}=\sum_{i=1}^{N} q_{i}$ and $\lambda=E\left(\lambda_{N}\right)$, then

$$
\begin{aligned}
\left|\mathrm{S}_{N}(x)-\mathrm{NB}_{\widehat{n}, \widehat{p}}(x)\right| & \leq \min \left\{\frac{1-e^{-\lambda}}{\lambda}, \frac{1}{x}\right\}\left(\frac{\lambda^{2}}{\widehat{n}-\lambda}+E\left|\lambda_{N}-\lambda\right|\right) \\
& +\min \left\{E\left(\frac{1-e^{-\lambda_{N}}}{\lambda_{N}} \sum_{i=1}^{N} \frac{q_{i}^{2}}{p_{i}}\right), \frac{1}{x} E\left(\sum_{i=1}^{N} \frac{q_{i}^{2}}{p_{i}}\right)\right\},
\end{aligned}
$$

where $\mathrm{S}_{N}(0)=E\left(\prod_{i=1}^{N} p_{i}\right)$.

Proof. Let $\mathrm{P}_{\lambda}(x)$ be the Poisson probability function with mean $\lambda$. It follows the fact that

$$
\left|\mathrm{S}_{N}(x)-\mathrm{NB}_{\widehat{n}, \widehat{p}}(x)\right| \leq\left|\mathrm{S}_{N}(x)-\mathrm{P}_{\lambda}(x)\right|+\left|\mathrm{P}_{\lambda}(x)-\mathrm{NB}_{\widehat{n}, \widehat{p}}(x)\right| .
$$

Teerapabolarn [2] and [1] showed that

$$
\begin{aligned}
\left|\mathrm{S}_{N}(x)-\mathrm{P}_{\lambda}(x)\right| & \leq \min \left\{\frac{1-e^{-\lambda}}{\lambda}, \frac{1}{x}\right\} E\left|\lambda_{N}-\lambda\right| \\
& +\min \left\{E\left(\frac{1-e^{-\lambda_{N}}}{\lambda_{N}} \sum_{i=1}^{N} \frac{q_{i}^{2}}{p_{i}}\right), \frac{1}{x} E\left(\sum_{i=1}^{N} \frac{q_{i}^{2}}{p_{i}}\right)\right\}
\end{aligned}
$$

and

$$
\left|\mathrm{P}_{\lambda}(x)-\mathrm{NB}_{\widehat{n}, \widehat{p}}(x)\right| \leq \min \left\{\frac{1-e^{-\lambda}}{\lambda}, \frac{1}{x}\right\} \frac{\widehat{n} \widehat{q}^{2}}{\widehat{p}}=\min \left\{\frac{1-e^{-\lambda}}{\lambda}, \frac{1}{x}\right\} \frac{\lambda^{2}}{\widehat{n}-\lambda},
$$


respectively. Hence, the inequality (2.1) is obtained by putting the right hand side of (2.3) and (2.4) to (2.2).

If $X_{i}$ 's are identically distributed, then the following corollary is an immediately consequence of the Theorem 2.1

Corollary 2.1. For $x \in \mathbb{N}$, if $p_{1}=p_{2}=\cdots=p$, then we have the following:

$$
\begin{aligned}
\left|\mathrm{S}_{N}(x)-\mathrm{NB}_{\widehat{n}, p}(x)\right| \leq & \min \left\{\frac{1-e^{-\widehat{n} q}}{\widehat{n} q}, \frac{1}{x}\right\}\left(\frac{\widehat{n} q^{2}}{p}+E|N-\widehat{n}| q\right) \\
& +\min \left\{E\left(1-e^{-N q}\right), \frac{\widehat{n} q}{x}\right\} \frac{q}{p},
\end{aligned}
$$

where $\mathrm{s}_{N}(0)=E\left(p^{N}\right)$.

\section{Examples}

This section, we give two examples to illustrate the result in the case of $X_{i}$ 's are identically distributed, which is in the Corollary 2.1 .

Example 3.1. For $n(n \in \mathbb{N})$ is fixed, let $N$ be a positive integer-valued random variable with probability function

$$
P(N=k)= \begin{cases}\frac{1}{2} & , k=n \\ \frac{1}{2} & , k=2 n \\ 0 & , \text { otherwise }\end{cases}
$$

Therefore $\widehat{n}=\frac{3 n}{2}, E|N-\widehat{n}|=\frac{n}{2}$ and $E\left(1-e^{-N q}\right)=\frac{2-e^{-n q}-e^{-2 n q}}{2}$. Let $p_{1}=p_{2}=\cdots=p$, then we have

$$
\begin{aligned}
& \left|\mathrm{s}_{N}(x)-\mathrm{NB}_{\frac{3 n}{2}, p}(x)\right| \leq \min \left\{\frac{1-e^{-\frac{3 n q}{2}}}{\frac{3 n q}{2}}, \frac{1}{x}\right\}\left(\frac{3 n q^{2}}{2 p}+\frac{n q}{2}\right) \\
& +\min \left\{2-e^{-n q}-e^{-2 n q}, \frac{3 n q}{x}\right\} \frac{q}{2 p},
\end{aligned}
$$

where $x \in \mathbb{N}$.

Example 3.2. Let $N$ be a positive integer-valued random variable with probability function

$$
P(N=n)=\frac{1}{2^{n}}, n=1,2, \ldots,
$$


then we have $\widehat{n}=2$ and $E|N-\widehat{n}|=1$. If $p_{1}=p_{2}=\cdots=p$, then we obtain

$$
\left|\mathrm{S}_{N}(x)-\mathrm{NB}_{2, p}(x)\right| \leq \min \left\{\frac{1-e^{-2 q}}{2 q}, \frac{1}{x}\right\}\left(\frac{2 q^{2}}{p}+q\right)+\min \left\{1, \frac{2 q}{x}\right\} \frac{q}{p},
$$

where $x \in \mathbb{N}$.

\section{Conclusion}

In this study, a pointwise bound for the point metric between the distribution of random sums of independent geometric random variables and an appropriate negative binomial distribution with parameters $\widehat{n}$ and $\widehat{p}$ cloud be obtained. In view of this bound, it is pointed out that the probability function of random sums of independent geometric random variables can be approximated by the negative binomial probability function with parameters $\widehat{n}$ and $\widehat{p}$ when $\widehat{q}=1-\widehat{p}$ is small.

\section{References}

[1] K. Teerapabolarn, A pointwise approximation for independent geometric random variables, Int. J. Pure Appl. Math., 76 (2012), 727-732.

[2] K. Teerapabolarn, A pointwise Poisson approximation for random sums of geometric random variables, Int. J. Pure Appl. Math., 89 (2013), 353-356.

[3] P. Vellaisamy and N. S. Upadhye, Compound negative binomial approximations for sums of random variables, Probab. Math. Statist., 29 (2009), 205-226. 